\title{
Estratégias de educação em saúde para idosos: experiências e desafíos
}

\author{
Estrategias de educación en salud para ancianos: \\ experiencias y desafíos \\ Health education strategies for older people: \\ experiences and challenges
}

Rodrigo Euripedes da Silveira ${ }^{1}$, Francielle Toniolo Nicodemos Furtado de Mendonça ${ }^{2}$, Álvaro da Silva Santos ${ }^{3}$, Elvira Maria Ventura Filipe ${ }^{4}$

\begin{abstract}
${ }^{1}$ Enfermeiro. Mestre em Ciências da Saúde. Doutorando em Ciências pela Coordenadoria de Controle de Doenças da Secretaria do Estado da Saúde (SES) de São Paulo. rodrigoeuripedes.silveira@gmail.com

${ }^{2}$ Enfermeira. Especialista em Saúde do Adulto na modalidade Residência Multiprofissional. Mestranda do Programa de PósGraduação em Atenção à Saúde da UFTM. francielletoniolo.enf@gmail.com

${ }^{3}$ Enfermeiro. Especialista em Saúde Pública. Mestre em Administração em Serviços de Saúde. Doutor em Ciências Sociais. Pós

Doutorado em Serviço Social. Professor Adjunto III de Graduação e da Pós Graduação Estrito Senso em Atenção à Saúde da Universidade Federal do Triângulo Mineiro (UFTM). alvaroenf@hotmail.com

${ }^{4}$ Psicóloga. Doutora em Psicologia da Saúde. Docente do Programa de Pós Graduação Estrito Senso em Ciências da Coordenadoria de Controle de Doenças da SES - São Paulo.elvira@crt.saude.sp.gov.br

Cómo citar este artículo en edición digital: Silveira, R.E., Mendonça, F.T.N.F., Santos, A.S. y Filipe, E.M.V. (2015). Estratégias de educação em saúde para idosos: experiências e desafíos. Cultura de los Cuidados (Edición digital), 19, 42. Disponible en: http:// dx.doi.org/10.14198/cuid.2015.42.14>

Correspondencia: Rodrigo Eurípedes da Silveira. Rua Campos Sales, 840. Bairro Abadia. Uberaba, Minas Gerais, Brazil. CEP: 38026-260. Tel.: +55(34) 9155-3088

Correo electrónico: rodrigoeuripedes.silveira@gmail.com

Recibido: 12/01/2015; Aceptado: 08/06/2015
\end{abstract}

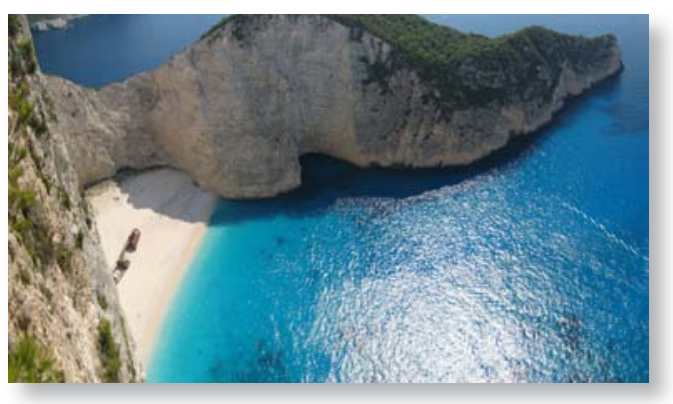

\section{ABSTRACT}

This is an integrative review which aims to assess the scientific literature related to health education for elderly in Brazil, between 2007 to 2013. How to search databases used the Virtual Health Library - VHL, Scielo, Redalyc and from the intersection of descriptors: aged and health education. Rose 14 articles that responded to the goals and, that after reading it he elucidated three categories, namely: Experiences in health education with elderly people,
Experiences of training and capacity-building Needs related to health education and physical activity as a tool for health education for the elderly. It turns out that one of the main gaps are the extension of studies and experiences with didactic approaches further, the appreciation of aspects beyond diseases, as well as the importance of physical activity, but also issues such as leisure, income generation, family relationships, in addition to better training of health professionals to deal with health education activities for the elderly.

Keywords: Elderly. Health Education. Elderly Health.

\section{RESUMEN}

Esta es una revisión integrativa que pretende evaluar las publicaciones científicas relacionadas con la educación de salud para an- 
cianos en Brasil entre 2007-2013. Cómo bases de busqueda de datos se utilizó la Biblioteca Virtual en Salud - BVS, Scielo, Redalyc y con los descriptores: educación en salud y anciano. Se evidenciarón 14 artículos que respondieron a los objetivos y, que después de leerlos se há dilucidado tres categorías, a saber: experiencias en educación para la salud con personas mayores, experiencias de formación y necesidades de capacitación relativas con educación para la salud y la actividad física como una herramienta para la educación de salud para los ancianos. Las principales necesidades de investigación y experiencias son la ampliación de estudios y experiencias con enfoques didácticos más lejos, la apreciación de los aspectos más allá de enfermedades, así como la importancia de la actividad física, pero también temas como la generación de ingresos, el ocio, las relaciones familiares, además de mejor formación de los profesionales de salud para hacer frente a las acciones de educación en salud para ancianos.

Palabras clave: Anciano. Educación en Salud. Salud del Anciano.

\section{RESUMO}

Esta é uma revisão integrativa que tem como objetivo avaliar a produção científica relacionada à educação em saúde para idosos no Brasil, entre 2007 a 2013. Como bases de busca se usaram a Biblioteca Virtual em Saúde - BVS, Redalyc e Scielo, a partir do cruzamento dos descritores: idoso e educação em saúde. Se levantou 14 artigos que responderam aos objetivos e, que após leitura se elucidou três categorias, a saber: Experiências em Educação em Saúde com Idosos, Experiências de Formação e Necessidades de Capacitação relacionadas à Educação em Saúde e Atividade Física como instrumento para Educação em Saúde para Idosos. Verifica-se que dentre as principais lacunas está à ampliação de estudos e experiências com abordagens didáticas mais amplas, a valorização de aspectos para além de doenças, bem como a importância da atividade física, mas também de questões como lazer, geração de renda, relações familiares, além de melhor capacitação de profissionais de saúde para lidar com ações de educação em saúde para idosos.

Palavras-Chave: Idoso. Educação em Saúde. Saúde do Idoso.

\section{INTRODUÇÃO}

Em um conceito abrangente, as ações educativas em saúde constituem processos dinâmicos de interação e aprendizagem entre profissionais de saúde, gerentes e outros, tendo como pano de fundo a busca pela melhoria das condições de saúde de determinada população. Destaca-se que esta modalidade de intervenção no âmbito da saúde coletiva, tem se desenvolvido significativamente nos últimos anos, com enfoque em ações preventivas e de promoção da saúde (Pinafo, Nunes, González, 2012).

Neste sentido, a abordagem multiprofissional no contexto da Educação em Saúde é de grande importância, para o alinhamento de programas, metas e práticas, desenvolvidas pelos profissionais de saúde entre si, com a instituição e, sobretudo, com o usuário, procurando desencadear mudanças de comportamento individual. Estas ações procuram modificar as condições de vida das pessoas, auxiliando no processo de tomada de decisão em direção à qualidade de vida e à saúde (Melo et al., 2009).

Contudo, as práticas educativas no contexto da Atenção Primária são perpetradas por dificuldades e desafios para sua devida execução. Na visão de enfermeiros, existem dificuldades no contexto do processo de trabalho 
da equipe, barreiras relacionadas à estrutura física e insuficiência de recursos materiais nas unidades de saúde. Além disso, no relato dos sujeitos, observam-se desencontros na relação com os usuários no desenvolvimento da educação em saúde, sobretudo em grupos que requerem abordagens e condutas específicas, como a condução de Grupos de Idosos (Moutinho et al, 2014).

Ao se considerar uma população crescente de pessoas com 60 anos ou mais de idade, consolida-se um desafio para os sistemas de saúde e seguridade social, que devem oferecer suporte de qualidade de vida para uma população com mais de 32 milhões de idosos, na sua maioria de nível sócio-econômico e educacional baixo e com alta prevalência de doenças crônicas e incapacitantes (Tavares, Dias, Munari, 2012). De fato, é imperativo o desenvolvimento de políticas sociais e de saúde factíveis e condizentes com as reais necessidades desta população, fugindo do modelo tradicional de atenção ao idoso, limitado às enfermidades crônicas em consultas individuais esporádicas, sem continuidade, e desconsiderando o impacto desse quadro na qualidade de vida (Melo et al., 2009).

Uma das ações propostas para o envelhecimento bem-sucedido é a educação em saúde. Para que as pessoas possam vivenciar um envelhecimento bem-sucedido, é fundamental construir a melhor relação possível entre quantidade e qualidade de vida. A Organização Mundial da Saúde (OMS) definiu o termo "envelhecimento ativo" como processo de otimização das oportunidades de saúde, participação e segurança, para expressar o decurso de conquista do envelhecimento como experiência positiva (Buranello et al., 2014).

Nesta direção, capacitar os profissionais de saúde com apoio e supervisão de gestores ap- tos para esta abordagem é premente para a eficácia destas estratégias. Em estudo que buscou avaliar as contribuições da prática educativa na qualificação de enfermeiros para o cuidado a idosos hospitalizados, pontuou-se que o idoso requer cuidados diferenciados e a prática educativa constitui-se em instrumento de qualificação e atualização primordial para os integrantes da equipe de enfermagem (Both et al., 2014).

Outro estudo, que considerou prontuários dos idosos participantes de um grupo de assistência multidisciplinar, identificou na comparação entre os resultados obtidos das avaliações fisioterapêuticas inicial e final, diminuição do tempo de execução do teste de sentar e levantarem 91,66\% da amostra, manutenção dos escores encontrados na avaliação da mobilidade, e melhora da pontuação na avaliação do equilíbrio em 54,16\% da amostra, havendo significância estatística $(\mathrm{p}<0,05)$. Experiências desta monta, assinalam a necessidade de inclusão das atividades de Educação em Saúde para idosos na agenda de cuidados das unidades de Atenção Primária à Saúde de todo país (Silva et al., 2014).

Levando em conta os temas supracitados, a presente investigação tem como objetivo avaliar a produção científica relacionada à educação em saúde para idosos no Brasil, entre 2007 a 2013, através de revisão integrativa da literatura.

\section{METODOLOGIA}

Trata-se de um estudo de revisão integrativa; com busca realizada nas seguintes fontes de dados: Biblioteca Virtual em Saúde - BVS, Redalyc e Scielo, a partir do cruzamento dos seguintes descritores: idoso e educação em saúde. Os limites de refinamento consideraram: artigos originais e completos oriundos de 
trabalhos desenvolvidos no Brasil, nos idiomas português, espanhol e inglês, publicados entre 2007 a 2013. Foram excluídos artigos duplicados, editoriais, opiniões e resenhas/capítulos de livro.

Em consideração aos critérios de seleção propostos, do universo de 324 artigos encontrados, 67 foram filtrados para leitura, dos quais quatorze constituíram a amostra final desta investigação. Os artigos foram tabulados e processados com análise descritiva, através das seguintes variáveis: o banco ou base de dados onde foram extraídos os trabalhos, ano de publicação, identificação dos autores, objetivos, palavras-chave, referencial teórico, metodologia aplicada e contribuições dos estudos. Posteriormente os dados foram categorizados por aproximação temática, inerente ao enfoque da presente investigação.
73,3\% das publicações, seguido por Educação em Saúde em 66,7\% e Promoção à Saúde em $53,3 \%$.

Com relação ao delineamento metodológico dos estudos, $50 \%$ dos estudos tinham abordagem qualitativa e outros $42,9 \%$ eram relato de experiência, como se observa no quadro 1. Entre os autores, 53,4\% eram enfermeiros, vinculados majoritariamente a departamentos e instituições de saúde ou educação. As instituições a que pertenciam estavam em sua maioria localizadas na região sudeste, denotando maior produção científica neste eixo, sobretudo nos estados de Rio de Janeiro (31,8\%) e São Paulo (13,6\%).

Quadro 1 - Distribuição dos artigos sobre Educação em Saúde para Idosos, quanto ao ano de publicação, periódico, título e tipo de estudo/método. Brasil, 2007-2013.

\section{RESULTADOS}

\section{Quatro periódicos} abarcaram o maior número de publicações sobre a temática no período estudado. São eles: Acta Paulista de Enfermagem, Ciência e Saúde Coletiva, Revista Brasileira de Enfermagem e Texto Contexto em Enfermagem, cada uma com duas publicações.

Das publicações do período, houve predomínio de publicações nos anos de 2009 e 2010, com quatro publicações cada uma, que somados representam $57,1 \%$. Foi registrada uma publicação em 2013. Com relação aos descritores, o termo Idoso aparece em

\begin{tabular}{|c|c|c|c|}
\hline Ano & Periódico & Título & Tipo de estudo/método \\
\hline 2007 & O Mundo da Saúde & $\begin{array}{l}\text { Ações educativas em promoção da saúde } \\
\text { no envelhecimento: } \\
\text { a experiência do núcleo de atenção ao } \\
\text { idoso da UNATI/UERJ }\end{array}$ & Relato de experiência \\
\hline 2007 & $\begin{array}{l}\text { Texto Contexto } \\
\text { Enferm }\end{array}$ & $\begin{array}{c}\text { Necessidades de Educação em Saúde dos } \\
\text { Cuidadores de Pessoas Idosas no } \\
\text { Domicilio }\end{array}$ & $\begin{array}{c}\text { Estudo exploratório- } \\
\text { descritivo, com abordagem } \\
\text { qualitativa }\end{array}$ \\
\hline 2008 & $\begin{array}{l}\text { Ciência \& Saúde } \\
\text { Coletiva }\end{array}$ & $\begin{array}{l}\text { A formação de profissionais para a atenção } \\
\text { integral à saúde } \\
\text { do idoso: a experiência interdisciplinar do } \\
\text { NAI - UNATI/UERJ }\end{array}$ & Relato de experiência \\
\hline 2009 & $\begin{array}{l}\text { Texto Contexto } \\
\text { Enferm }\end{array}$ & $\begin{array}{l}\text { Cuidado compartilhado: uma perspectiva } \\
\text { de cuidar do idoso fundamentada na } \\
\text { educação em saúde }\end{array}$ & $\begin{array}{l}\text { Pesquisa qualitativa de } \\
\text { abordagem convergente- } \\
\text { assistencial }\end{array}$ \\
\hline 2009 & Rev. APS & $\begin{array}{l}\text { A saúde no diálogo com a vida cotidiana: a } \\
\text { experiência do trabalho educativo com } \\
\text { idosos no Grupo Roda da Saúde. }\end{array}$ & Relato de experiência \\
\hline 2009 & Rev Bras Enferm & $\begin{array}{l}\text { Necessidades de saúde do idoso: } \\
\text { perspectivas para a enfermagem }\end{array}$ & Método qualitativo \\
\hline 2010 & $\begin{array}{l}\text { Interface - } \\
\text { Comunic., Saude, } \\
\text { Educ }\end{array}$ & $\begin{array}{l}\text { Educação em saúde da pessoa idosa em } \\
\text { discursos e práticas: atividade fisica como } \\
\text { sinônimo de saúde. }\end{array}$ & $\begin{array}{c}\text { Estudo } \\
\text { empirico de cunho qualitativo }\end{array}$ \\
\hline 2010 & $\begin{array}{l}\text { Psicologia \& } \\
\text { Sociedade }\end{array}$ & $\begin{array}{l}\text { ‘Grupos de conversa': saúde da pessoa } \\
\text { idosa na estratégia saúde da família. }\end{array}$ & Relato de experiência \\
\hline 2010 & $\begin{array}{l}\text { Revista Brasileira } \\
\text { de Atividade Física } \\
\text { \& Saúde }\end{array}$ & $\begin{array}{l}\text { Dança e envelhecimento: } \\
\text { uma parceria em movimento! }\end{array}$ & Relato de experiência \\
\hline 2011 & $\begin{array}{l}\text { Rev. Bras. Geriatr. } \\
\text { Gerontol. }\end{array}$ & $\begin{array}{c}\text { Atividade Física para idosos: diretrizes } \\
\text { para implantação de } \\
\text { programas e ações. }\end{array}$ & Pesquisa do tipo qualitativa \\
\hline 2012 & Acta Paul Enferm. & $\begin{array}{c}\text { Alfabetização em saúde de pessoas idosas } \\
\text { na atenção básica }\end{array}$ & Pesquisa do tipo qualitativa \\
\hline 2012 & Escola Anna Nery & $\begin{array}{l}\text { Reinventando práticas de enfermagem na } \\
\text { educação em saúde: teatro com idoso. }\end{array}$ & Pesquisa do tipo qualitativa \\
\hline 2012 & Acta Paul Enferm. & $\begin{array}{c}\text { Qualidade de vida de idosos e participação } \\
\text { em atividades } \\
\text { educativas grupais }\end{array}$ & $\begin{array}{l}\text { Estudo tipo inquérito } \\
\text { domiciliar transversal, tipo } \\
\text { quantitativo }\end{array}$ \\
\hline 2013 & $\begin{array}{l}\text { Revista Eletrônica } \\
\text { Gestão \& Saúde }\end{array}$ & $\begin{array}{l}\text { Oficinas multiprofissionais: educação em } \\
\text { saúde para idosos de uma comunidade }\end{array}$ & Relato de experiência \\
\hline
\end{tabular}




\section{DISCUSSÃO}

Os artigos foram analisados, codificados e tabulados em três categorias temáticas, com o intuito de elucidar a síntese das informações emergentes sob o aspecto do conteúdo e corroborar na construção do conhecimento sobre o tema pesquisado, a saber: Experiências em Educação em Saúde com Idosos, Experiências de Formação e Necessidade de Capacitação relacionadas à Educação em Saúde e Atividade Física como instrumento para Educação em Saúde para Idosos.

\section{1) Experiências em Educação em Saúde com}

\section{Idosos}

Nesta categoria foram incluídos os artigos que explicitam diferentes formas de se fazer educação em saúde com idosos, demonstrando uma diversidade de técnicas e formas de abordagens que podem ser utilizadas tanto na atenção primária como na atenção hospitalar. Em experiência realizada por um grupo de residentes no Rio Grande do Sul com quarenta idosos participantes de um grupo de convivência, foram trabalhados os temas: osteoporose, diabetes mellitus, dislipidemia, planejando o futuro e relações de cuidado. Pela perspectiva dos autores, as oficinas contribuíram de modo inovador para estimular a participação dos idosos, promovendo a interação e o convívio dos mesmos, enquanto assumiam o papel de agentes ativos no processo de aprendizagem e de fazer saúde (Serbim, 2013).

No Rio de Janeiro a experiência de um programa vinculado à Universidade Aberta da Terceira Idade incluiu diferentes categorias profissionais e várias modalidades educativas, abertas aos idosos e usuários em geral. $\mathrm{Na}$ iniciativa o trabalho pautou-se em uma visão de saúde articulada à qualidade de vida e concebeu a educação como diálogo, troca de experiências, respeito ao outro, reflexão, problematização e busca de alternativas/escolhas possíveis. $\mathrm{O}$ projeto contribuiu no estímulo a uma nova sensibilidade na cultura profissional e representou um "abrir portas" no serviço, fortalecendo o compromisso com uma assistência de qualidade, centrada no vínculo, na responsabilização e na partilha dos desafios à qualidade de vida e saúde no envelhecimento (Assis et al., 2007).

Outro artigo proveniente da mesma instituição apresentou a proposta metodológica do grupo aberto Roda da Saúde, que se caracterizou como "chá da tarde" e buscou estimular a reflexão e a produção coletiva sobre temas relacionados à saúde e qualidade de vida com programação definida no próprio grupo, utilizando temas emergentes no processo e/ou sugeridos pelos participantes. A experiência tem sido valiosa para profissionais e idosos pela partilha de saberes e construção de vínculos (Bernardo et al. 2009).

Em estudo realizado no Mato Grosso do Sul são relatados resultados parciais do desenvolvimento de uma experiência de extensão universitária realizada em parceria com a rede de atenção primária. As atividades realizadas buscaram desenvolver um processo grupal de promoção da saúde com pessoas idosas, tendo em vista o envelhecimento ativo e a qualidade de vida dessa população. Foi observado que as atividades em grupo constituíram-se em espaço privilegiado para a constituição de redes de apoio e de processo de ensino-aprendizagem, possibilitando aos participantes a ampliação do conhecimento de si e do outro, por meio de vivências e discussão em grupo (Combinato et al. 2010).

Em experiência realizada pela Universidade de Pernambuco o teatro foi utilizado como ferramenta de educação em saúde, ten- 
do como enfoque a promoção da saúde num grupo de idosos. Nos encontros foram trabalhados os temas: acolhimento, construção das histórias de vida, retirando máscaras, viajando na imaginação/diversidade dos cenários, trabalhando a emoção, trabalho corporal, fazendo caras e bocas, oficina de personagens e ensaiando uma peça teatral - "O que a vida me ensinou e o que posso lhe adiantar". O teatro se mostrou excelente instrumento de empoderamento da população idosa, a partir da valorização de suas experiências de vida (Campos et al., 2012).

Estratégia realizada no Rio de Janeiro utilizou a educação em saúde pautada no diálogo como veículo de cuidado. O trabalho foi realizado com 19 acompanhantes de idosos hospitalizados que participaram de entrevistas e observações participantes buscando identificar os saberes e práticas sobre os cuidados na prevenção e tratamento de úlceras por pressão, integrá-los a um plano de cuidados e discutir sua efetividade. $\mathrm{Na}$ análise do plano de cuidado inferiu-se que o diálogo/reflexão permitiu ao acompanhante se instrumentalizar, e ofereceu condições para pensar, refletir criticamente e agir, em busca da participação no cuidado. Assim, demonstrou-se que estratégias de educação em saúde também podem ser implementadas de forma eficaz no cenário hospitalar (Teixeira; Ferreira, 2009).

Em estudo realizado com 1255 idosos que teve como objetivo comparar os escores de qualidade de vida entre os idosos participativos e não participativos de atividades educativas grupais, encontrou-se que os idosos que participavam de atividades educativas grupais apresentaram melhores escores no domínio relações sociais e nas facetas funcionamento dos sentidos, autonomia, atividades passadas, presentes e futuras e participação social, com- parados aos não participativos. Dessa forma os autores evidenciaram no estudo a necessidade de reflexão sobre as atividades educativas grupais desenvolvidas nos serviços de saúde como fator contributivo para a melhoria das condições de saúde e qualidade de vida dos idosos (Tavares; Dias; Munari, 2012).

Em consideração à temática ora abordada, refere-se à diversidade de técnicas didáticas existentes em outras áreas não diretamente ligadas à saúde, mas que posem ser adaptadas para grupos de idosos no contexto da atenção primária. Pesquisas e experiências que avancem além do debate sobre doenças, que perpassem o limite do tempo e alcancem maior interesse do usuário, bem como as técnicas que possam avaliar o impacto das ações desenvolvidas e também práticas educativas em saúde na área hospitalar parecem constituir lacunas a serem melhor exploradas na educação em saúde para idosos.

\section{2) Experiências de Formação e Necessidade de Capacitação relacionadas à Educação em Saúde}

A prática profissional de saúde, sobretudo em níveis específicos de atendimento, permite observar uma desarticulação entre os conteúdos teóricos absorvidos na graduação e aqueles utilizados na prática, em que a condução de grupos de Educação em Saúde muitas vezes não alcança seus reais objetivos. Com a população idosa não é diferente, uma vez que os grupos direcionados a essas pessoas na Atenção Primária, muitas vezes, têm sua finalidade reduzida à verificação da pressão arterial e de glicemia capilar aliada à entrega de medicamentos. Este contexto reitera a necessidade de capacitação e orientação dos profissionais de saúde para a condução destas atividades.

Estudo realizado em Santa Catarina com 


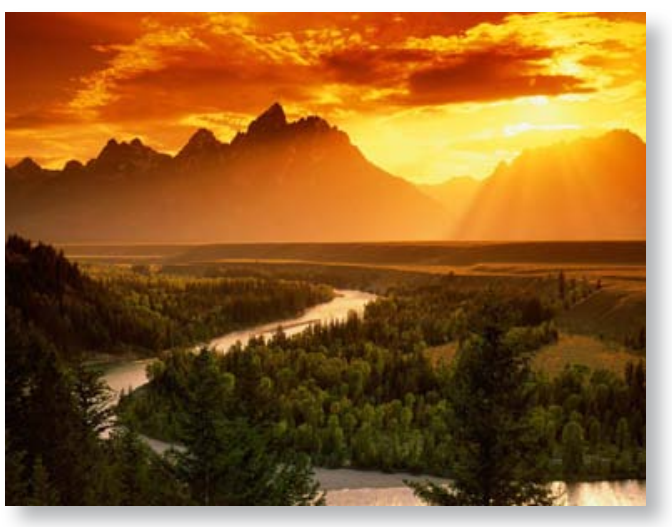

abordagem qualitativa e com vistas a identificar e classificar as necessidades de educação em saúde apresentadas por cuidadores de idosos, observou que as necessidades educativas mais prementes estavam relacionadas às doenças e seus agravos, à terapia medicamentosa, dietas e exercícios físicos (Martins et al, 2007).

Por outra perspectiva, estudo conduzido com treze usuários de uma Unidade Básica de Saúde (UBS) do Rio de Janeiro, questionou aos idosos quais eram as suas expectativas com relação à enfermagem. Emergiram como categorias: Encontrar o outro (humano) na atitude da enfermagem e estabelecer relações com outras pessoas. Entre as ações não práticas da enfermagem preferidas pelos idosos predominaram: Ajudar a resolver problemas, Bater papo, Dar orientação e Passear juntos (Lima e Tocantins, 2009).

A investigação conduzida no estado do Rio Grande do Sul, buscou analisar como pessoas idosas vinculadas a grupos de educação em saúde de uma UBS buscam, compreendem e partilham informações a fim de manter e promover a saúde ao longo da vida. Pelos relatos dos 30 idosos, foram elaboradas cinco categorias de análise - interesse/preocupação em saúde; busca, compreensão partilha e repercussão das informações para o idoso. Contudo, muitos idosos destacaram que as informações recebidas eram bastante restritas, referentes ao diagnóstico e ao tratamento, enquanto as dúvidas relatadas eram mais amplas e não esclarecidas, demonstrando que a educação em saúde das pessoas idosas necessita ser aprofundada (Paskulin et al, 2012).

Destaca-se que a capacitação de profissionais para atuar na área de envelhecimento e saúde do idoso é uma das ações prioritárias da Política Nacional do Idoso no Brasil, em função do acelerado envelhecimento populacional e da consequente transição epidemiológica (incremento das doenças crônicas não transmissíveis) do país. Desta forma, refere-se à iniciativa do Núcleo de Atenção ao Idoso, vinculado à Universidade Aberta da Terceira Idade/UERJ, que desenvolve um programa de ensino a partir da experiência assistencial, cujo eixo compreende a integralidade da atenção e o trabalho interdisciplinar. O programa inclui modalidades de ensino em nível de residência, especialização, treinamento profissional e estágio de graduação. (Motta, Caldas e Assis, 2008).

Outro conceito em expansão no Brasil se refere aos preceptores, entendidos como um componente emergente do sistema de saúde brasileiro para educação de recursos humanos. Em pesquisa com participantes de distintas áreas profissionais em um programa educacional como preceptores, foram registradas quarenta e três competências, classificadas em nove domínios: educação, valores profissionais, ciências básicas da saúde pública, gestão, saúde, trabalho em equipe, comunicação, comunidade orientação e desenvolvimento profissional. Essas competências constituem uma referência útil para educação profissional continuada (Rodrigues, Witt; 2013).

Aspectos voltados à necessidade de capacitação dos profissionais para lidarem com idosos através das práticas de educação em saúde precisam ser mais exploradas, pois além de poucos estudos parecem que os profissionais 
"ousam pouco" na criatividade para lidar com conteúdos dos idosos. Seja na universidade na formação básica, ou mesmo em capacitações continuadas, as técnicas didáticas, temas de saúde (mais do que os de doença) precisam ser mais explorados, o que constituem também importantes lacunas.

\section{3) Atividade Física como instrumento para}

\section{Educação em Saúde para Idosos}

No entendimento de um processo natural relativo ao envelhecimento, admite-se a presença de alterações físico-funcionais que podem levar à redução ou incapacidade de realização de atividades físicas ou da rotina diária, coexistindo com fraqueza muscular, alterações no equilíbrio e no balance e modificações na mobilidade, que corroboram a um aumento no risco de quedas, depressão e hospitalizações (Buranello et al., 2014).

É neste contexto que se discute a antiga percepção do idoso enquanto um "peso" para a sociedade, que passa a contemplar o envelhecimento como um processo repleto de oportunidades, passível de uma vida saudável e com qualidade de vida. Nesta direção, estudo que envolveu 14 idosos frequentadores de Academias da Terceira Idade e sete profissionais de saúde responsáveis pela implementação e coordenação de ações de educação e promoção da saúde para a Terceira Idade em um município de Santa Catarina, verificou-se que os profissionais dão significado a atividade física como principal estratégia para a promoção de uma "velhice saudável" e que os discursos dos idosos reproduzem, em grande medida, essas construções. (Fernandes, Siqueira, 2010).

Contudo, cabe considerar que, em países cuja distribuição de renda se caracteriza por forte desigualdade, posição em que se encontra o Brasil, graves "patologias sociais", advindas de políticas públicas de baixa efi- cácia, ações de saúde pautadas em alicerces curativistas, entre outros fatores, produzem corpos frágeis, enfermos e vulneráveis (Castiel e Alvarez-Dardet, 2007). Nesta monta, a adoção exclusiva da prática de exercícios físicos como principal estratégia para a promoção de um envelhecimento saudável, não é válida, uma vez que outras dimensões essenciais para a vida plena e saudável, que incluem aspectos culturais, condições básicas de vida que dizem respeito à moradia, trabalho, lazer e acesso aos serviços de saúde, também devem ser foco das abordagens em saúde pública.

Estudo realizado em Santa Catarina, com o intuito de descrever as diretrizes necessárias para a formulação de programas e ações de atividade física (AF) para idosos de grupos de convivência, observou que, para melhorar e ampliar a estratégia é necessário: descentralização, contratação de profissionais especializados, conscientização sobre um envelhecimento ativo e os benefícios da prática regular de atividade física, além da necessidade do estabelecimento de parcerias com instituições públicas e privadas (Salin et al, 2011). Esta experiência deve ser levada em conta na elaboração e implementação de estratégias que favoreçam a prática de atividade física orientada, além da supervisão nas denominadas Academias da Terceira Idade alocadas em praças, parques e outros espaços públicos.

Além disso, outra estratégia de grande interesse para grupos de idosos refere-se à Dança, que permite a seus participantes trabalhar a capacidade funcional por meio de coreografias, exigindo assim orientação têmporo-espacial, entre outras possibilidades. O Programa de Atividade Física para Terceira Idade - PROFIT da Universidade Estadual Paulista utiliza a dança como atividade regular desde 1997 e passou por reestruturações que favoreceram a dinamização do programa e o alcance de me- 
lhores resultados. Na turma de 30 alunos, além do trabalho motor, dos ritmos variados e montagem coreográfica, as aulas propiciam a estes idosos, consciência corporal, prática mental e ritmo. As aulas são dividas em alongamento e aquecimento inicial, parte principal (onde são estimulados os componentes da capacidade funcional) e finalizam com alongamento e volta à calma (Paiva et al, 2011).

Para além disso, um cuidado especializado nos setores primários de saúde devem incluir ações de saúde que estejam ligadas as doenças mais prevalentes; diagnosticar os riscos de doenças e prevenir as internações; traçar o perfil dos idosos e suas reais necessidades; assim como outras questões inerentes ao envelhecimento populacional brasileiro, tais como: geração de renda, relações familiares, lazer e recreação, que constituem outras lacunas de pesquisa e intervenção educativa em saúde com idosos (Silveira et al, 2013).

\section{CONSIDERAÇÕES FINAIS}

Pelos resultados obtidos por esta análise da produção científica em Educação em Saúde direcionada à população idosa, é possível inferir que se faz necessário um enfoque na integralidade da atenção e no cuidado em aspectos relevantes para esta população, como as questões nutricionais, de atividade física e de lazer, que devem ser trabalhados enquanto objetivos da prevenção e promoção de saúde nos diversos níveis de atenção, sobremaneira na Atenção Primária, porta de entrada para o Sistema de Saúde brasileiro.

Ressalta-se que é essencial e estratégico a multiplicação de oportunidades de formação nesta linha de cuidado em face às demandas sociais crescentes pelo envelhecimento populacional. De mesma relevância, considera-se o apoio informal e familiar na consecução de um aspecto fundamental na atenção à saúde desse grupo populacional. Diversas estratégias podem ser utilizadas para tais objetivos, tais como: distribuição de cartilhas e folhetos do Ministério da Saúde e Secretarias Estaduais e Municipais de Saúde; o desenvolvimento de campanhas em programas populares de rádio; veiculação de propagandas na televisão; treinamento de agentes comunitários de saúde e profissionais integrantes da estratégia de saúde da família para, no trabalho domiciliar, estimular os cidadãos na adoção de comportamentos saudáveis.

O fato é que os idosos precisam lançar mão de algumas estratégias que possibilitem ter uma velhice satisfatória. Isto, implica em estarem atentos à cultivar novos hábitos, engajar-se em atividades produtivas, realizar seus projetos de vida, ingressar em universidade de terceira idade, desenvolver serviços voluntários, dentre tantas outras iniciativas possíveis.

Um envelhecimento bem-sucedido depende de como o idoso vai enfrentar os desafios da vida, lutar pelos os seus direitos de cidadão e colocar em prática projetos viáveis dentro das condições pessoais e do meio ambiente em que vivem. Reforçar essa concepção do papel do enfermeiro como educador e agente de transformação social, portanto, se faz necessária. Esta deve ser uma constante na atuação profissional, visto que a educação permanente em saúde deve ser parte integrante do escopo profissional.

\section{REFERÊNCIAS}

- Assis, M., Pacheco, L.C., Menezes, M.F.G., Bernardo, M.H.J., Steenhagen C.H.V.A., Tavares, E.L. y Santos, D.M. (2007). Ações educativas em promoção da saúde no envelhecimento: a experiência do núcleo de atenção ao idoso da UNATI/UERJ. O mundo da Saúde, 31, 438-47.

- Bernardo, M.H.J., Menezes, M.F.G., Assis, M., Carvalho, L.C., y Mecenas, A.S. (2009). A saúde no diálogo com a vida cotidana: a experiência do trabalho educativo com 
idosos no grupo roda da saúde. Revista APS, 12(4), 504-9.

- Both, J.E., Leite, M.T., Hildebrandt, L.M., Beuter, M., Muller, L.A., y Linck, C.L. (2014). Qualificação da equipe de enfermagem mediante pesquisa convergente assistencial: contribuições ao cuidado do idoso hospitalizado. Esc. Anna Nery Rev. Enferm., 18, 486-95.

- Buranello, M.C., Gomes, N.C., Patrizzi, L.J., Farinelli, M.R., Shimano, S.G.N. (2014). Grupo de Ginástica Orientada na Terceira Idade: condições funcionais e adesão. Rev. Fam. Ciclos de Vida e Saúde no Cont. Soc,. 2, 13-9.

- Campos, C.N.A., Santos, L.C., Moura, M.R., Aquino, J.M. y Monteiro, E.M.L.M. (2012). Reinventando práticas de enfermagem na educação em saúde: teatro com idosos. Esc. Anna Nery Rev. Enferm., 16, 588-96.

- Castiel, L.D., y Alvarez-Dardet, C. (2007). A saúde persecutória: os limites da responsabilidade. Rio de Janeiro: Fiocruz.

- Combinato, D.S., Vecchia, M.D., Lopes, E.G. Manoel, R.A., Marino, H.D., Oliveira, A.C.S., y Silva, K.F. (2010). "Grupos de Conversa”: saúde da pessoa idosa na estratégia saúde da família. Psicologia \& Sociedade, 22, 558-568.

- Fernandes, W.R. y Siqueira, V.H.F. (2010). Health education for elderly people in discourse and practice: physical activity as a synonym for health. Interface - Comunic., Saude, Educ., 14, 371-385.

- Lima, C.A. y Tocantins, F.R. (2009). Necessidades de saúde do idoso: perspectivas para a enfermagem. Rev. Bras. Enferm., 62, 367-373.

- Martins, J.J., Albuquerque, G.L., Nascimento, E.R.P., Barra, D.C.C., Souza, W.G.A. y Pacheco, W.N.S. (2007). Necessidades de educação em saúde dos cuidadores de pessoas idosas no domicílio. Texto Contexto Enferm, 16, 254-262.

- Melo, M.C., Souza, A.L., Leandro, E.L., Mauricio, H.A., Silva, I.D., y Oliveira, J.M.O. (2009). A educação em saúde como agente promotor de qualidade de vida para o idoso. Ciência \& Saúde Coletiva., 14, 1579-1586.

- Motta, L.B., Caldas, C.P., y Assis, M. (2008). A formação de profissionais para a atenção integral à saúde do idoso: a experiência interdisciplinar do NAI-UNATI/UERJ. Ciência \& Saúde Coletiva. 13, 1143-51.

- Moutinho, C.B.,Almeida, E.R., Leite, M.T.S., y Vieira,
M.A. (2014). Dificuldades, desafios e superações sobre educação em saúde na visão de enfermeiros de saúde da família. Trab. educ. saúde, 12, 253-72.

- Paiva, A.C.S., Hernandez, S.S., Sebastião, E., Quadros-Junior, A.C., Cury, M., Costa, J.L.R,y Gobbi, L.T.B., y Gobbi, S. (2011). Dança e envelhecimento: uma parceria em movimento! Rev. Brasil. Ativ. Fís. Saúde, 15, 70-2.

- Paskulin, L.M.G., Bierhals, C.C.B.K., Valer, D.B., Aires, M., Guimarães, N.V., Brocker, A.R., Lanziotti, L.H. y Morais, E.P. (2012). Alfabetização em saúde de pessoas idosas na atenção básica. Acta Paul. enferm. 25, 129-35.

- Pinafo, E. y Nunes, E.F.P.A. y González, A.D. (2012). A educação em saúde na relação usuário-trabalhador no cotidiano de equipes de saúde da família. Ciência \& Saúde Coletiva, 17, 1825-32.

- Rodrigues, C.D.S. y Witt, R.R. (2013). Competencies for preceptorship in the Brazilian health care system. J Contin Educ Nurs., 44, 507-15. Recuperado de: doi: 10.3928/00220124-20130903-63.

- Salin, M.S., Mazo, G.Z., Cardoso, A.S. y Garcia, G.C. (2011). Atividade Física para idosos: diretrizes para implantação de programas e ações. Rev. Bras. Geriatr. Gerontol; 14, 197-208.

- Serbim, A., Gerlack, L., Motta, M.D., Gaviolli, C., Cecconello, M., Moreira, L. y Werlang, M. (2012). Oficinas multiprofissionais: educação em saúde para idosos de uma comunidade. Gestão e Saúde, 4, 1780-90.

- Silva, T.O., Glisoi, S.F.N., Ansai, J.H. y Izzo, H. (2014). Grupo de assistência multidisciplinar ao idoso ambulatorial: efeitos da abordagem fisioterapêutica. Rev Soc Bras Clin., 12, 2-7.

- Silveira, R.E., Santos, A.S., Monteiro, T.S.A. y Sousa, M.C. (2013). Hospitalización de ancianos y el trabajo de enfermería: Análisis de la producción científica brasileña. Cultura de los Cuidados, 36, 77-88.

- Tavares, D.M.S., Dias, F.A. y Munari, D.B. (2012). Qualidade de vida de idosos e participação em atividades educativas grupais. Acta Paulista de Enfermagem, 25, 601-6.

- Teixeira, M.L.O. y Ferreira, M.A. (2009). Cuidado compartilhado: uma perspectiva de cuidar do idoso fundamentada na educação em saúde. Texto \& Contexto - Enfermagem, 18, 750-8. 